\title{
El beneficio de las redes académicas en el desarrollo de la excelencia en la educación superior del Ecuador
}

\section{The benefit of academic networks in the development of excellence in higher education in Ecuador}

Ing. Com. Víctor Hugo Moscoso Zamora, MSc

Erika Sonia Quiñonez Alvarado

Piedad Ysidora Vera Franco

Betilde Josefina Contreras Caicedo

Universidad de Guayaquil, Ecuador

Autor para correspondencia: victor.moscosoz@ug.edu.ec

Fecha de recepción: 21 de Noviembre de 2016 - Fecha de aceptación: 10 de Enero de 2017

Resumen: Las Redes y colectivos académicos, son un pilar fundamental en la búsqueda de la Excelencia Académica de la Educación Superior. Ecuador, busca mejorar y desarrollar la calidad educativa, para lograrlo, fue imperativo hacer reformas a la Constitución de la República, y las normativas académicas; las primeras integraciones entre Universidades Públicas y Privadas del país dieron sus frutos y rompieron paradigmas inclusos culturales, que se plasman en este documento, mostrando que mediante consenso nacional se lograron elaborar diseños y rediseños curriculares, que generaron nuevas y mejores ofertas académicas que están siendo aprobadas por el Consejo de Educación Superior Ecuatoriano (CES), esto condujo hacia la excelencia académica y el logro de la calidad educativa, entre otros beneficios, que servirán de referentes empíricos para posteriores estudios; además, permitirá la movilidad estudiantil y docente, en un inicio a nivel nacional y a mediano plazo Internacional.

Palabras Claves: Redes académicas; Excelencia; Calidad; Movilidad

Abstract: Networks and collective academic, are a mainstay in the pursuit of Academic Excellence in Higher Education, Ecuador, seeks to improve and develop the quality of education, to achieve this, it was imperative to reform the Constitution of the Republic, and academic regulations ; the first integrations between public and private universities in the country paid off and broke or not cultural paradigms, which are reflected in this document, showing that through national consensus achieved elaborate designs and curriculum redesign, generating new and better academic offerings being approved by the Higher Education Council of Ecuador (CES), this led to academic excellence and achievement of educational quality, among other benefits, which will serve as empirical references for further study; It will also allow the student and faculty mobility, initially at national and international medium term.

Keywords: Academic networks; Excellence; Quality; Mobility 


\section{Introducción}

Ecuador, país sudamericano en vías de desarrollo, en esta última década, realiza cambios, genera estrategias y crea lineamientos para el logro de nuevas y mejores metas, en búsqueda de una Educación Superior de Excelencia, uno de los pilares que le permitirá el desarrollo sustentable y sostenible del mismo. Para forjarlo se tornó imperativo reformar, la Constitución de la República 2008, la Ley Orgánica de Educación Superior (LOES) 2010 y el Reglamento de Régimen Académico (RRA) 2013, siendo esta última, la normativa que regula y orienta el que hacer académico de las Instituciones de Educación Superior (IES).

En este artículo se busca recopilar las experiencias de la integración de instituciones de educación superior de algunos zonas de Ecuador (ver apéndice B), para concebir un diseño curricular que permita el intercambio de estudiantes, docentes e investigadores; detallar los procesos que realizaron las comisiones conformadas, para crear el macro, meso y micro currículo; y exponer el resultado final (ver apéndice A). Describe el esfuerzo aunado y comprometido de las universidades, que rompió barreras culturales y geográficas en pro de una estructura de currículo, con sustentación metodológica fundamentada en las leyes y normativas.

En relación con lo anterior, se resalta que uno de los objetivos específicos de estas redes universitarias, era la elaboración de diseños curriculares que permitan y faciliten la movilidad nacional e internacional de estudiantes, profesores, investigadores, y profesionales, es decir de una universidad a otra, con miras a la Integración de la Comunidad Académica Ecuatoriana en la dinámica del conocimiento a nivel regional y mundial, establecidos en el Reglamento de Régimen Académico (RRA).

\section{Metodología}

Esta investigación es de tipo descriptivo porque se realizará un detalle del proceso de integración de 26 Instituciones de Educación Superior durante el periodo 2014 - 2016 (Ver apéndice C), para desarrollar un Diseño Curricular acorde a las exigencias del Reglamento de Régimen Académico del Ecuador vigente, esto es, que incluyan los conocimientos disciplinares, interdisciplinares, transdisciplinares, profesionales, investigativos, de saberes integrales y de comunicación, necesarios para elaborar una estructura curricular, y por ende, construir el perfil del futuro profesional integrando las necesidades del contexto laboral actual y la pertinencia de las carreras. Los datos serán narrados como fueron observados (Arias, 2012), con el fin de exponer, con criterio analítico, los resultados de las primeras experiencias y perspectivas nacionales en la formación y desarrollo de las Redes, y Colectivos Académicos, entre las Universidades Públicas y Privadas del Ecuador.

El diseño de la investigación es documental y bibliográfica con enfoque cualitativo, se realiza una recopilación, revisión y análisis de datos secundarios como textos, leyes y reglamentos de fuentes impresas y electrónicas, que soportan el proceso realizado en la concepción del currículo (Arias, 2012). Es importante destacar que este artículo, puede servir como un referente empírico, para la continuidad de otras investigaciones relacionadas o correlacionadas. 


\section{Antecedentes}

Inicialmente se podría establecer que el término, Red Académica, se refiere a "una estructura que dispone de un patrón que la caracteriza" (Porto \& Gardey, 2014), y un espacio en el que se encuentra información relevante y actualizada, necesaria para las comunidades educativas y la comunicación en los procesos de enseñanza aprendizaje.

Según el artículo 102 del RRA, este tipo de redes podrán constituirse para efectos del diseño y ejecución de programas o proyectos de investigación, o de vinculación con la sociedad en factor de suma importancia, puesto que, la producción de conocimientos por parte de la Universidad, tienen que tener como objetivo principal el lograr beneficios reales para la sociedad.

Algunos autores como; Peter Haas (1989), citado en artículo por Alma Maldonado (2005), propone el termino Comunidades Epistémicas "red de profesionales con reconocida experiencia y competencia en un campo particular", equivalente a lo que se conoce cómo Colectivos Académicos, término que se utiliza en el Reglamento de Régimen Académico vigente del Ecuador, para describir al conglomerado de profesores e investigadores en diferentes áreas del conocimiento de una o varias unidades académicas pertenecientes a la misma o diversas Instituciones de Educación Superior, para promover el debate intelectual, el diseño de proyectos de investigación, y procesos de autoformación establecidos en el artículo 100 del RRA.

Se debe señalar que la Lógica Epistémica, se desarrolla recientemente, pero como "un campo de la lógica modal que se ocupa del razonamiento del conocimiento, contrastes epistemológicos que buscan el desarrollo de las ciencias" (Dogan \& Parhe, 1993: 80): cuya aplicación, se da en diversas áreas del conocimiento, y que han sido de gran utilidad, pues, enriquecen a la comunidad científica, y por ende a la academia, convirtiéndose en "universidades invisibles o redes de personas con ideas afines" (Crane, 1972).

Basado en lo anteriormente expuesto, y los referentes teóricos consultados, se infiere que no puede concebirse una Universidad carente o deficiente de investigación científica, por lo que se expresa la necesidad de la conformación de colectivos académicos integrado por profesionales expertos o redes académicas, que busquen la producción de conocimiento, la vinculación y el beneficio social.

Ecuador, inicia un proceso de depuración y mejoramiento de la calidad en la Educación Superior inicialmente con el Mandato Constituyente No. 14, expedido por la Asamblea Nacional Constituyente el 22 de julio de 2008, que estableció la obligación del Consejo Nacional de Evaluación y Acreditación (CONEA) de elaborar un informe técnico sobre el nivel de desempeño institucional de los establecimientos de educación superior, a fin de garantizar su calidad.

Este Mandato constituyente, fue una iniciativa orientada a recuperar el rol director, regulador y supervisor del Estado, sobre las instituciones de educación superior, cuyo análisis estableció las deficiencias de las universidades ecuatorianas, pues, se evidencia, a lo largo del informe, "un conjunto fragmentado por múltiples distanciamientos en lo académico, 
investigativo y tecnológico, brechas que, a su vez, expresan múltiples fenómenos" (CONEA, 2009), por lo que se establecen nuevos retos para el sistema de educación superior, que se plasman en las resoluciones de las normativas correspondientes, las que se reforman conforme a las necesidades y exigencias del contexto.

La búsqueda de la calidad y el logro de la excelencia en la academia ecuatoriana, se evidencian en indicadores propuestos por los organismos de control de la educación superior, y se consideran de mucha relevancia especialmente: El indicador de desarrollo de contextos pedagógicos y curriculares interactivos, creativos y de construcción innovadora del conocimiento; por ende, el indicador docente, ya que, desde allí debe emanar la transmisión del conocimiento, y la responsabilidad de la formación del perfil del futuro profesional acorde a las necesidades del contexto y del mercado laboral.

Todo esto conllevó a la imperiosa necesidad de conformar comunidades científicas, mediante la integración de colectivos académicos, y redes entre universidades nacionales, con la finalidad de iniciar un proceso de autoformación que nos permita enfrentar los nuevos retos de calidad de la Universidad Ecuatoriana, y en un principio el diseño de carreras nuevas y rediseño o reestructuración de carreras vigentes. Ofertas académicas enmarcadas y acordes a las normativas actuales.

A partir de Agosto del 2014, se toman las primeras iniciativas por parte de Universidades Publicas y Privadas de integrarse en redes académicas de manera progresiva, con el objetivo básico de tratar de bosquejar la nueva estructura curricular, es decir, organizar prospectivamente los conocimientos que serían necesarios para desarrollar el perfil profesional y académico del estudiante conforme a las exigencias del Reglamento de Régimen Académico vigente, y las verdaderas necesidades del contexto laboral, esto fue un gran reto desde el inicio, puesto que, habría primero que consensuar criterios, aspecto que complicaba la integración de Universidades de diferentes zonas del país, por el factor cultural (costa y sierra) y las necesidades del desarrollo de la zona (situación geográfica), incluso de orgullo personal.

\section{Proceso de Integración de Universidades para el Diseño Curricular}

Se hace un sumario de las fases que comprendieron el desarrollo del currículo, proceso en el que intervinieron delegados de 26 universidades del Ecuador (ver apéndice C), tanto públicas como privadas, en un esfuerzo mancomunado por conseguir la excelencia académica sobre bases sostenibles, desde la perspectiva de uno de los involucrados en esta integración.

\section{Construcción de la Estructura Curricular}

La meta inicial no implicaba la estandarización de mallas curriculares para cada oferta académica en el Ecuador, sino más bien, homologar y actualizar contenidos, y así facilitar la movilidad de estudiantes y docentes de una Institución de educación superior a otra que oferte la misma carrera; también actualizar e interpretar nuevos conocimientos y enriquecer la universidades ecuatorianas, mediante el aporte de cada representante o delegado de las mismas, para la construcción del nuevo Macro, Meso y Micro currículo que se propondría en cada 
carrera, y que tendría como base, la estructura determinada por la norma vigente el Reglamente de Régimen Académico.

Esta labor fue compleja, analítica y critica desde lo sistemático, académico y epistémico, pues, habría que considerar e involucrar para su elaboración; actores de la sociedad y de los sectores productivos, ejes de desarrollo, objetivos estratégicos además de considerar las planificaciones de la Secretaria Nacional de Planificación y Desarrollo (SENPLADES), y articular los objetivos de las carreras al Plan Nacional del Buen Vivir (PNBV), y a las agendas zonales, manteniendo una correlación con el modelo pedagógico y ecológico de las Universidades del país.

\section{Elaboración del Macro Diseño}

Para el desarrollo del macro currículo, se organizaron mesas de trabajo conformadas por pedagogos, sociólogos, académicos e investigadores delegados por las 26 Universidades entre Públicas y Privadas integradas en la Red, y cuyo objetivo básico fue como primer paso determinar la Pertinencia que debería tener la nueva oferta académica, pilar fundamental en la construcción de esta nueva estructura, puesto que se deberán establecer, determinar, analizar, identificar, vincular y articular aspectos que a continuación se detallan:

a) Análisis de Problemas y necesidades del contexto y objetivos del PNBV, que abordará la profesión.

b) Determinar los Horizontes Epistemológicos presentes en la profesión.

c) Identificar los núcleos básicos de las disciplinas que sustentan la profesión.

d) Vinculación de las tecnologías de punta a los aprendizajes profesionales a fin de garantizar la respuesta a los problemas que resolverá la profesión en los sectores estratégicos y de interés público.

e) Identificar problemas de la realidad (actores y sectores vinculados a la profesión) que integran el objeto de estudio de la profesión.

f) Establecer las tendencias de desarrollo local y regional que están incluidas en los campos de estudio y de actuación de la profesión.

g) Los aportes que realizará el currículo a las necesidades de formación del talento humano, incluyendo el análisis de demanda ocupacional

h) Determinar en forma clara las funciones y roles de los escenarios laborales en los que actuarán los futuros profesionales.

Esta primera fase de recopilación y construcción de la información correspondiente, necesitó de una investigación documental y de campo donde se utilizó como herramienta la encuesta, que se realizó en el contexto zonal correspondiente a cada Universidad, esta etapa abarcó aproximadamente seis sesiones de arduas deliberaciones previas a acuerdos, luego de lo cual, se procedió a consensuar el documento que establecía la pertinencia de la nueva oferta académica en una plenaria que contó con la asistencia aproximadamente 80 delegados de las 26 Universidades integradas a la Red Académica (ver apéndice C).

Esta experiencia se consideró histórica, ya que era la primera vez que se pudo integrar y llegar a consensos académicos entre Instituciones de Educación Superior de la costa y sierra de 
nuestro país, logrando disminuir significativamente la brecha cultural, que incluso, por el factor geográfico, y de diversidad de criterios académicos y epistémicos ha existido en la Universidad Ecuatoriana.

\section{Planificación Curricular}

La tarea inicia con la determinación del objeto de estudio de la carrera, con la misma organización para el desarrollo de la misma, es decir, las mesas de trabajo conformadas por los delegados de cada Institución participante en la red, y para su construcción se debería ir respondiendo a ciertos cuestionamientos como son:

- ¿Qué se estudia o interviene en formación?

- ¿Qué se quiere transformar con la profesión?

- ¿Con que aplicaciones y orientaciones metodológicas se transformarán los problemas referidos a la profesión?

- ¿Cuáles son las orientaciones del conocimiento y los saberes que tiene en cuenta la construcción del Objeto de Estudio de la profesión?

La elaboración del documento que contenía la planificación curricular, fue otro reto epistémico que necesitó de cinco sesiones de trabajo, puesto que, la diversidad de criterios, algunos con bases teóricas clásicas y otros modernas, hicieron que se generen debates intelectuales, que enriquecieron el conocimiento de la academia previo a llegar a un consenso general.

Para complementar la espina dorsal del diseño curricular macro, se necesitó establecer y determinar, mediante la misma mecánica de trabajo: El enfoque de género e interculturalidad, Campos de estudios, Modelo de investigación, Modelo de prácticas pre profesionales, Metodología y ambientes de aprendizajes y los Componentes de Vinculación con la sociedad, elementos relevantes para la conformación de la estructura curricular exigida por la normativa vigente. Las mesas de trabajo, ya llevaron mayor experiencia en la elaboración del diseño de las nuevas ofertas académicas y los aportes se realizan más mesuradamente, lo que mejoró la construcción de los documentos que se someten a consenso en las plenarias correspondientes.

Esto es un conquista de la red, puesto que los colectivos académicos o también denominados comunidades epistémicas, se acoplaron y lograron eficientemente resultados que resuelven las necesidades de la academia ecuatoriana, experiencias que serán útiles en procesos similares, ya que cada quinquenio se deberá revisar, reformar o actualizar la estructura académica de cada carrera que se oferte, además, es un indicador de relevancia en las evaluaciones que nos hacen los organismos de control de la educación superior (CEAACES).

\section{Elaboración del Meso Currículo}

Se puede decir que básicamente, un meso currículo, es una malla que plasma de manera resumida todo el proceso articulado e integrado de formación académica y profesional (asignaturas) del estudiante de una carrera, esta se estructura por niveles y ejes de formación (Universidad de las Artes del Ecuador, 2013). 
El reglamento de régimen académico vigente en el Ecuador, en el artículo 21 establece como se debe estructurar la malla (Meso Currículo), indica que las unidades de organización curricular para las carreras de 3er nivel son; Unidad Básica, Unidad Profesional y Unidad de Titulación, y el artículo 29, describe los campos de formación que son: Fundamentos Teóricos, Praxis Profesional, Epistemología y Metodología de la Investigación, integración de saberes contextos y cultura y Comunicación y Lenguajes (Consejo de Educación Superior, 2013).

El estructurar este tipo de malla (estructura nueva), conllevó involucrar en su elaboración todo lo establecido en el macro currículo, por lo que se necesitó de un proceso metodológico descriptivo, analítico y correlacional fundamentalmente, así que, se conformaron las mesas de trabajo por áreas de conocimiento, y por unidades de organización curricular y campos de formación, lo que generó intensos debates académicos, y ajustes en los criterios iniciales.

Puesto que, habría que elaborar una malla curricular que se ajuste a la normativa correspondiente del R.R.A y articule a los objetivos, políticas lineamientos o metas del Plan Nacional del Buen Vivir, Agendas Zonales, Ejes estratégicos, entre otros planes de desarrollo nacional de la SENPLADES, considerar las necesidades del mercado laboral, establecer los núcleos problémicos a resolver y los resultados del aprendizaje que deberán ser evidenciados en un proyecto integrador de saberes en cada nivel.

Además, integrar las asignaturas propuestas en el nivel correspondiente, darles la secuencialidad y coherencia necesaria para lograr un proyecto integrador, y que, a su vez logre los resultados del aprendizaje, lo que a su vez, aporte a la construcción del perfil de egreso del futuro profesional, considerando cada unidad de organización curricular.

Fue en resumen, una labor bastante titánica para los expertos en cada disciplina básica que sustentan las diversas profesiones, incluso acoplar la distribución de la carga horaria acorde las actividades de aprendizaje estipuladas en los artículos 15,16 y 17 R.R.A.

Se considera que este tipo de experiencia intelectual ha sido única, puesto que es la primera vez que la Educación Superior de nuestro país, se somete a una reestructuración académica de esta magnitud, a fin de diseñar o rediseñar acorde a las nomenclaturas de la Clasificación Internacional Normalizada de la Educación (CINE) diseñadas por la Organización de las Naciones Unidas para la Educación, la Ciencia y la Cultura UNESCO, las carreras que actualmente oferta la universidad Ecuatoriana en la búsqueda de la excelencia académica.

\section{Elaboración del Micro Currículo}

Esta fue una labor más detallista, puesto que a pesar que conformarse las mesas de trabajo por áreas de conocimiento con los docentes expertos que imparten las asignaturas propuestas, se necesitaba construir una estructura de contenidos mínimos, previo a la construcción de los sílabos definitivos cuyo formato nuevo propuesto por el Consejo de Educación Superior (CES), involucra Objetivos, aspectos metodológicos, Unidades, Temas, subtemas, evaluación de aprendizaje, soportes teóricos, proyecto integrador entre otros. 


\section{La matriz debería contener:}
a) Nombre de la Asignatura
b) Unidad de Organización Curricular
c) Campo de formación
d) Resultados del Aprendizaje
e) Descripción mínima de los contenidos propuestos
f) Nivel o Periodo correspondiente
g) Distribución de las actividades de Aprendizaje (Horas)

\section{Convenios}

Las actividades de Practicas Pre profesionales y de Vinculación con la sociedad, forman parte de la estructura curricular a diseñar o rediseñar, en primer lugar fue necesario elaborar los reglamentos para el funcionamiento acorde de estas actividades, por ende, los convenios interinstitucionales son fundamentales para el que el estudiante pueda poner en práctica y beneficie a la sociedad con sus conocimientos, y la conformación de estas redes académicas generan la cooperación necesaria que facilite esta gestión académica, es decir, entre universidades se deben apoyar, además, fue importante la apertura para practicas pre profesionales, de ciertos entes gubernamentales, organismos de control y Gobiernos Autónomos Descentralizados GAD’s (regionales, provinciales, cantonales, parroquiales).

\section{Personal Docente}

El personal académico que impartirá las asignaturas propuestas, deberá tener a más de la experticia, un cuarto nivel (maestría) afín a la catedra, acorde a lo dispuesto por el reglamento de escalafón del Profesor e investigador, y lo dispuesto por el consejo de educación superior (CES), lo que obliga al docente entre otras cosas a actualizar sus conocimientos en forma continua, estos fueron temas que a pesar de que son muy internos de cada Universidad, eran de relevancia, reflexión y análisis, puesto que, las normativas son las mismas para todas las Universidades del país, además, se comenzó la planificación de Congresos Nacionales e internacionales en algunas de las Universidades integradas en la Red, a fin de incentivar a los docentes a elaborar Ponencias , e incluso a escribir Artículos Científicos, lo que mejora el nivel de los docentes para la re categorización y el indicador docente en la Acreditación Institucional.

\section{Infraestructura y Equipamiento}

El Consejo de Evaluación, Acreditación y Aseguramiento de la Calidad de la Educación Superior del Ecuador, tiene bien definido los parámetros e indicadores (Reglamentos) con relación a aulas, bibliotecas, equipamiento, entre otros, como manifesté, también fueron temas discutidos en sesiones de trabajo, y el objetivo buscaba la acreditación institucional, siempre con el análisis previo de la reglamentación respectiva, los docentes abogados fueron de mucha utilidad en la interpretación de los artículos respectivos, y en las plenarias para llegar a esclarecer lo exigido en estas normativas.

\section{Conclusiones}


La conformación de Redes y Colectivos Académicos en nuestro país, han permitido que mediante las comunidades epistémicas, se logren mejoras significativas en la Educación Superior Ecuatoriana, y consideramos que los resultados obtenidos preliminarmente por medio del trabajo en equipo, cooperación interinstitucional, aportes epistémicos y consensos, rompió las barreras académicas tradicionales, de criterios obsoletos e incluso paradigmas culturales y regionales en beneficio y crecimiento en calidad educativa, lo que permitiría se haga viable la movilidad estudiantil y Docente inicialmente entre nuestras Universidades, por lo que se consideró un gran paso para la consecución de las metas.

Paulatinamente cada Universidad integrada a la Red con esta base, ha elaborado nuevas y mejores ofertas académicas las cuales han sido propuestas al Consejo de Educación Superior (CES), y aprobadas para ponerlas en vigencia de forma inmediata, estos indicadores nos dan la pauta para buscar lograr la integración en Redes Internacionales, y pensar que un futuro muy cercano la movilidad podría ser internacional, consideramos entonces, que en verdad vamos en buen camino en la búsqueda de la Excelencia del Sistema de educación Superior Ecuatoriano.

\section{Recomendaciones}

Con el esfuerzo colectivo y comprometido de los actores sociales de Ecuador, en este caso las universidades que conformaron esta red académica, se puede lograr romper barreras que permitan avizorar un futuro prometedor hacia la excelencia y desarrollo de potencialidades de saberes, que ubiquen al país en el sitial mundial de formación de profesionales de alta calidad.

\section{Bibliografía}

Arias, F. G. (2012). El proyecto de investigación. Caracas: Episteme, C. A.

Asamblea Constituyente. (2008). Constitución de la República de Ecuador. Quito: Lexis.

Comisión Permanente de Universidades y Escuelas Politécnicas del CES. (2 de Mayo de 2014).

CES.gob.ec. Obtenido de CES.gob.ec: http://www.ces.gob.ec/doc/PlataformaPostgrados/formato-proyecto-carreras.pdf

Consejo de Educación Superior. (2013). Reglamento de Régimen Académico. Quito: Lexis.

Consejo de Educación Superior. (2016). Reglamento de Carrera y Escalafón del Profesor e Investigador del Sistema de Educación Superior (Codificación). Quito: LEXIS S. A.

Consejo de Educación Superior, CES. (2013). Plan Excelencia Universitaria.

Consejo de Evaluación, Acreditación y Aseguramiento de la Calidad de la Educación Superior, CEAACES. (2014). Reglamento de Evaluación, Acreditación y Categorización de Carreras de las Instituciones de Educación Superior. Quito: LEXIS S. A. 
Consejo Nacional de Evaluación y Acreditación de la Educación Superior del Ecuador, CONEA. (4 de Noviembre de 2009). Mandato Constituyente No. 14. Recuperado el 28 de Octubre de 2016, de Mandato Constituyente No. 14:

file://C:/Users/Erika/Downloads/2\%20informe\%20universidades\%20conea.pdf

Crane, D. (1972). Invisible Colleges. Diffusion of Knowledge in Scientific Communities. Chicago: Universtity Press.

Dogan, M., \& Pahre, R. (1993). Las nuevas ciencias sociales: La marginalidad creadora. UAMAzcapotzalco Solciológica, 80. Obtenido de http://www.revistasociologica.com.mx/pdf/2411.pdf

Elizabeth Larrea; Martha Montalván. (2016). Modelo Educativo Ecológico de la Universidad de Guayaquil. Guayaquil: Universidad de Guayaquil.

Granados, E. L. (20 de Abril de 2015). CES.gob.ec. Obtenido de CES.gob.ec: http://www.ces.gob.ec/doc/Taller-difusion/SubidoAbril-2015/curriculo_es-sistemico\%20$\% 20 \mathrm{e} \% 201$ arrea.pdf

Haas, P. (1989).

Maldonado, A. (2005). Comunidades epistémicas: una propuesta para estudiar el papel de los expertos en la definición de políticas en educación superior en México. Revista de la Educación Superior, 107-121. Obtenido de http://publicaciones.anuies.mx/pdfs/revista/Revista134_S4A5ES.pdf

Maldonado, A. (2005). Comunidades epistémicas: Una propuesta para estudiar el papel de los expertos en la definición de políticas en educación superior en México. Revista de la Educación Superior, 107-122.

Organización de las Naciones Unidas para la Educación, la Ciencia y la Cultura UNESCO.

(2011). uis.unesco.org. Obtenido de uis.unesco.org: http://www.uis.unesco.org/Education/Documents/isced-2011-sp.pdf Porto, J. P., \& Gardey, A. (2014). Definición. De. Obtenido de Definición. De: http://definicion.de/red-informatica/

Presidencia de la República. (2010). Ley Orgánica de Educación Superior. Quito: LEXIS S. A. Queipo, E. A., \& Buena fe, A. A. (25 de Octubre de 2016). INNOVA Research Journal. Obtenido de journaluidegye.com: http://www.journaluidegye.com/magazine/index.php/innova/article/view/108/76

Secretaría Nacional de Planificación y Desarrollo SENPLADES. (2013). Plan Nacional del Buen Vivir 2013 - 2017. Quito: Semplades. 
Subred de Contabilidad y Auditoría. (2016). Informes de la Sub red de Contabilidad y Auditoría 2014-2016.

Universidad de las Artes del Ecuador. (2013). Uartes.edu.ec. Obtenido de Uartes.edu.ec: http://www.uartes.edu.ec/descargables/resumen.pdf 


\section{Apéndices}

\section{Apéndice A: Diseño Curricular Contabilidad y Auditoría}

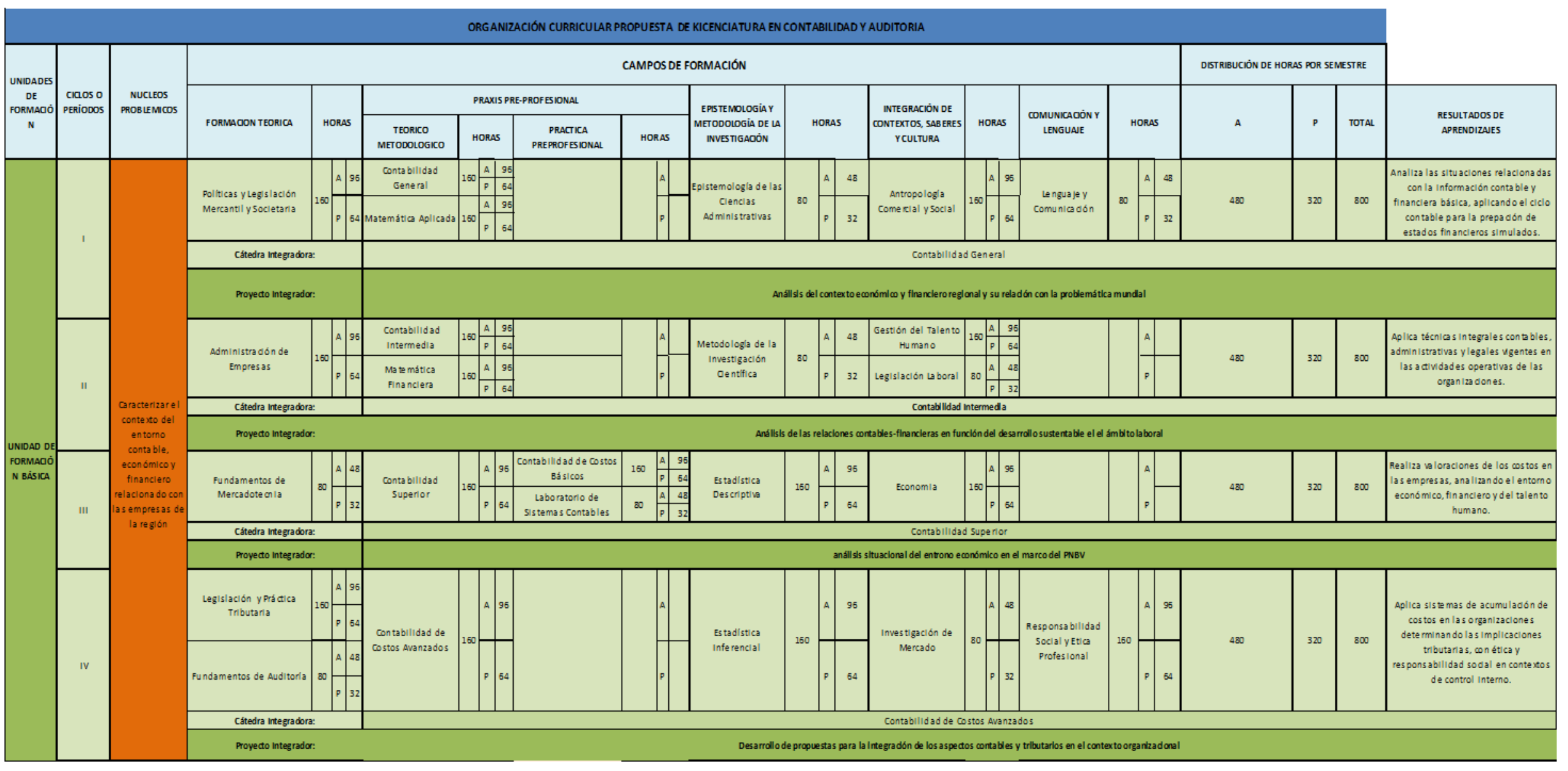

Figura A1. Meso Currículo, Unidad de Organización Básica. 


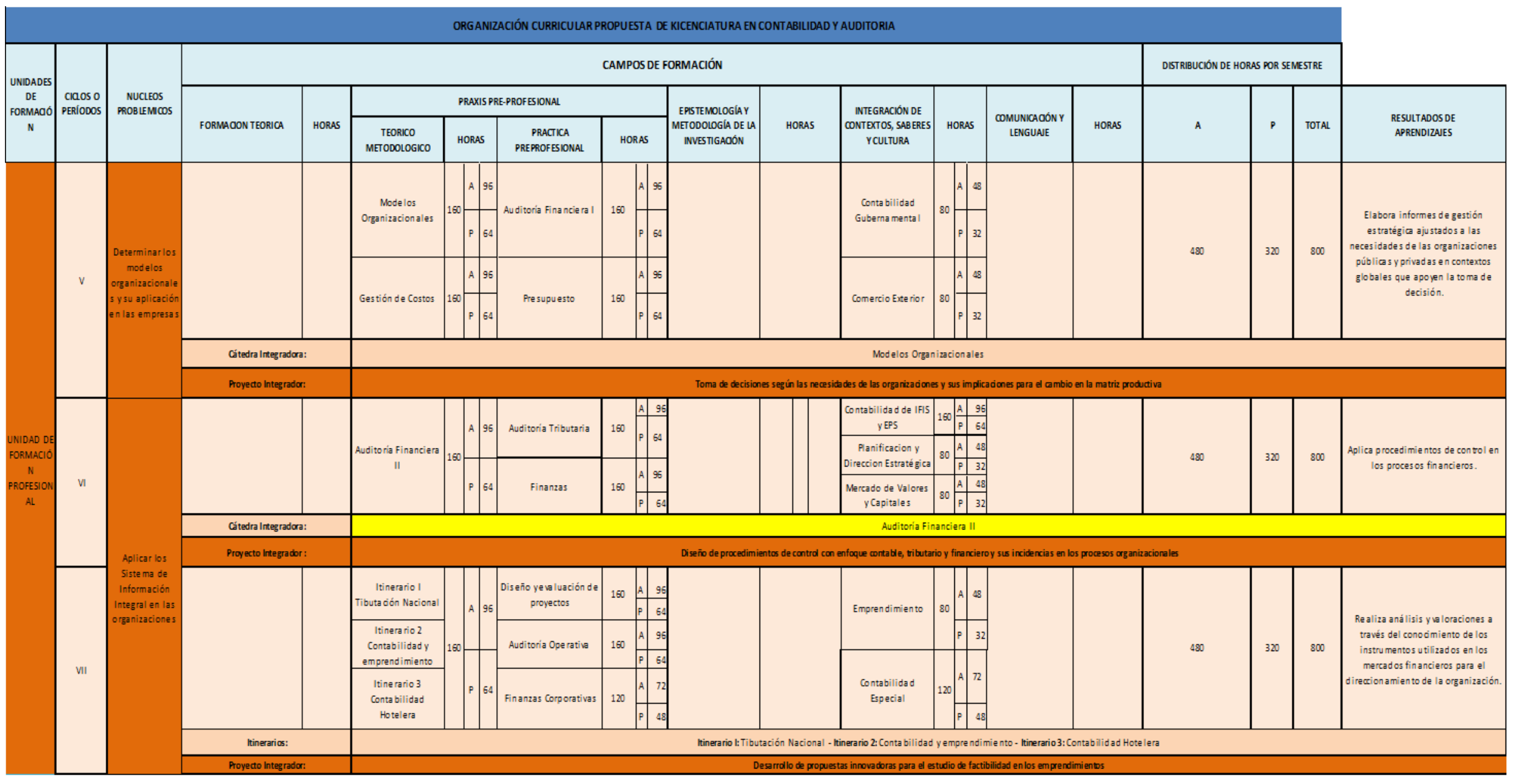

Figura A2. Meso Currículo, Unidad Organización Profesional. 


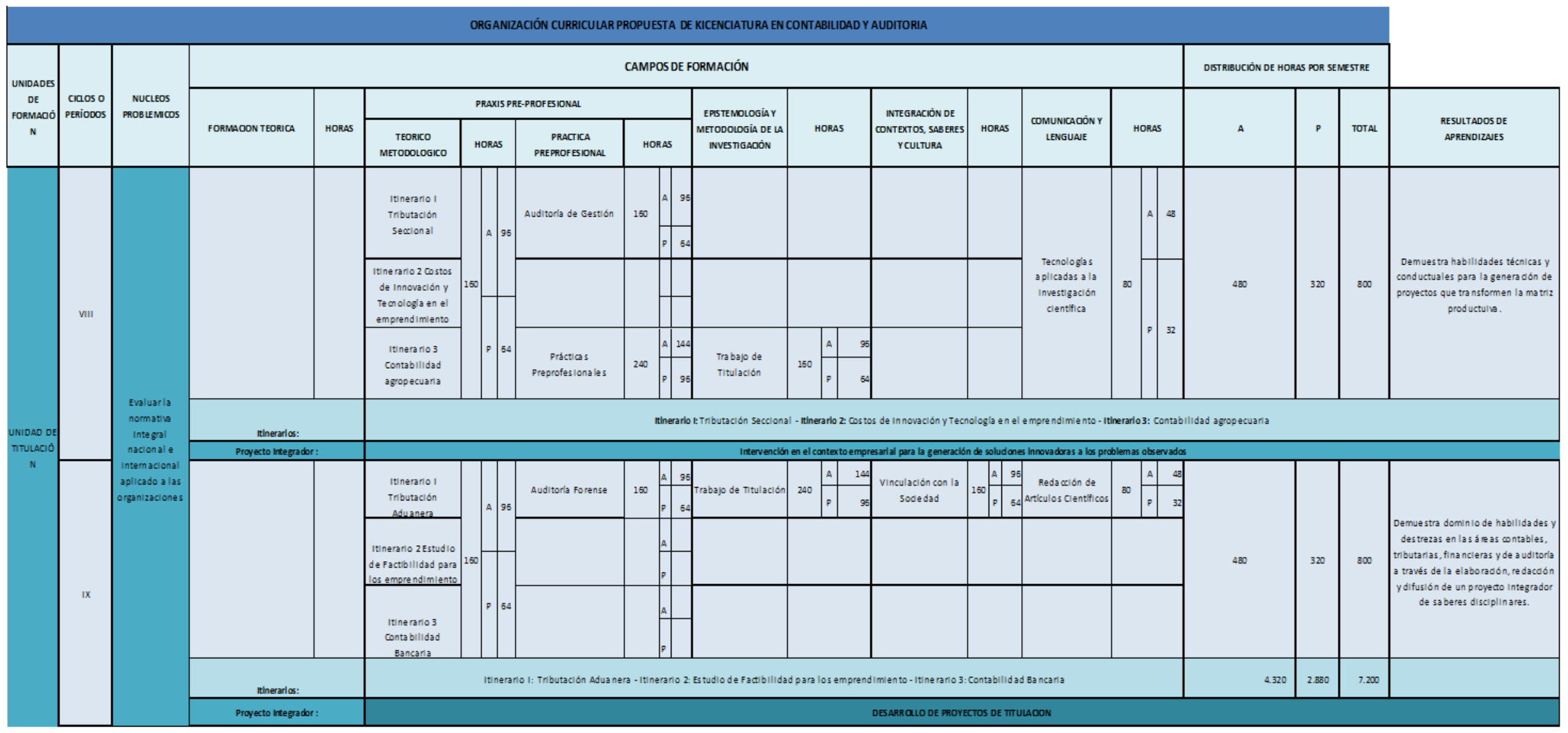

Figura A3. Unidad de Organización de Titulación. 


\section{Apéndice B: Zonas establecidas por la SENPLADES}

Zona 1:Provincias de: Esmeraldas, Imbabura, Carchi, Sucumbios.

Zona 2: Provincias de: Pichincha (excepto el cantón Quito), Napo, Orellana.

Zona 3: Provincias de Cotopaxi, Tungurahua, Chimborazo, Pastaza.

Zona 4: Provincias de: Manabí, Santo Domingo de los Tsáchilas.

Zona 5: Provincias de: Santa Elena, Guayas (excepto los cantones de Guayaquil Samborondóny Durán). Bolívar, Los Ríos y Galápagos.

Zona 6: Provincias de: Can̂ar, Azuay, Morona Santiago. Zona 7: Provincias de: El Oro, Loja, Zamora Chinchipe.

Zona 8: Cantones de: Guayaquil, Samborondón y Durán.

Zona 9: Distrito Metropolitano de Quito.

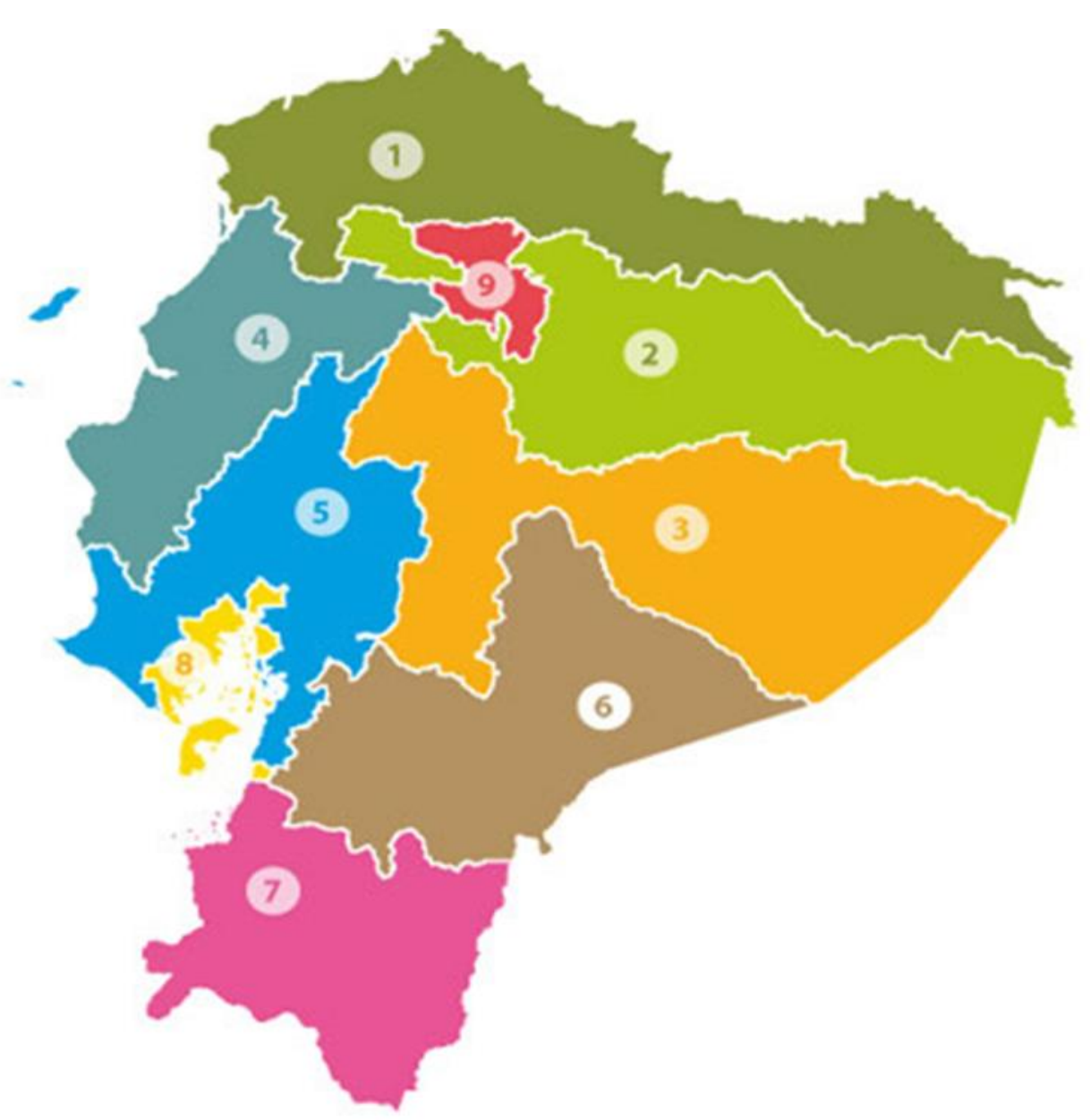

Figura B1: Distribución zonal. Tomado del Plan Nacional del Buen Vivir 2013-2107 (SENPLADES, 2013). 


\section{Apéndice C: Listado de Universidades Públicas y Privadas Integradas a la Sub red}

1) Universidad de Guayaquil (U.G)

2) Universidad Laica Vicente Rocafuerte (ULVR)

3) Universidad Católica Santiago de Guayaquil (UCSG)

4) Universidad Estatal de Milagro (UNEMI)

5) Universidad Estatal Península de Santa Elena (UPSE)

6) Universidad Técnica de Babahoyo (UTB)

7) Universidad Politécnica Salesiana (U.P.S)

8) Pontificia Universidad Católica del Ecuador (PUCE)

9) Universidad Técnica de Manabí (UTM)

10) Universidad Técnica Particular de Loja (UTPL)

11) Universidad Laica Eloy Alfaro de Manabí (ULEAM)

12) Escuela Superior Politécnica del Ejercito (ESPE)

13) Universidad Estatal del Sur De Manabí

14) Universidad Técnica de Machala (UTMACH)

15) Universidad Técnica de Cotopaxi (UTC)

16) Universidad Estatal de Bolívar

17) Universidad San Gregorio De Portoviejo

18) Universidad Técnica de Ambato (UTA)

19) Universidad Central del Ecuador (UCE)

20) Universidad Tecnológica Equinoccial (UTE)

21) Universidad Nacional de Chimborazo (UNACH)

22) Universidad Técnica Estatal de Quevedo (UTQ)

23) Universidad Católica de Cuenca (UCC) 
24) Universidad Tecnológica Indo américa (UTI)

25) Universidad del Azuay (UDA)

26) Universidad de Especialidades Espíritu Santo (UEES)

\section{Apéndice D: Logo}

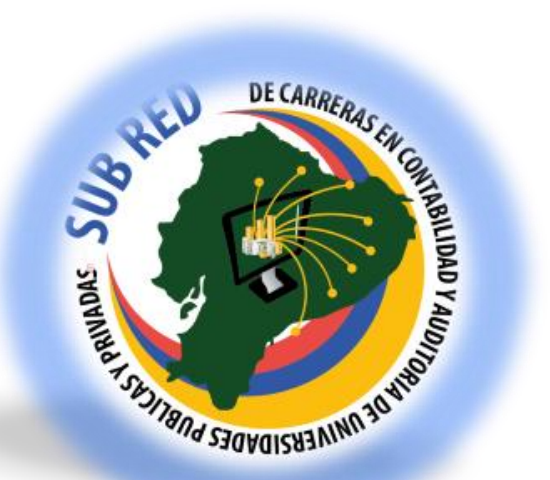

Figura D1: Tomado de (Subred de Contabilidad y Auditoría, 2016) 